\title{
1 Fluorescence recovery after photo-bleaching as a method to 2 determine local diffusion coefficient in the stratum corneum
}

3

\author{
Yuri G Anissimov ${ }^{1 *}$, Xin Zhao ${ }^{2}$, Michael S Roberts ${ }^{3}$, Andrei V Zvyagin ${ }^{2}$
}

${ }^{1}$ School of Biomolecular and Physical Sciences, Griffith University, Qld 4222, Australia

${ }^{2}$ MQ Biofocus Research Centre, Macquarie University, NSW 2109, Australia

${ }^{3}$ Therapeutics Research Centre, School of Medicine, University of Queensland, Australia

*Corresponding author: $\quad$ Tel.: +617 55528496; Fax +617 55528065,

E-mail: Y.Anissimov@griffith.edu.au

Keywords: Stratum corneum, lipids, diffusion coefficient, Rhodamine B, FRAP, modelling

\section{Abstract}

Fluorescence recovery after photo-bleaching experiments were performed in human stratum corneum in vitro. Fluorescence multiphoton tomography was used, which allowed the dimensions of the photobleached volume to be at the micron scale and located fully within the lipid phase of the stratum corneum. Analysis of the fluorescence recovery data with simplified mathematical models yielded the diffusion coefficient of small molecular weight organic fluorescent dye Rhodamine B in the stratum corneum lipid phase of about $(3 \sim 6) \times 10^{-}$ ${ }^{9} \mathrm{~cm}^{2} \mathrm{~s}^{-1}$. It was concluded that the presented method can be used for detailed analysis of localised diffusion coefficients in the stratum corneum phases for various fluorescent probes.

\section{Introduction}

The stratum corneum (SC) is the main barrier to drug delivery through skin and therefore understanding details of solute transport in SC is an important area of research. The SC is a complex multiphase membrane consisting of layers of corneocytes that are sealed tightly by densely packed lipid layers [1]. Fundamental parameters describing this transport are values of diffusion coefficients in different phases of the heterogeneous SC, that can be used in insilico models of the SC (e.g. [2, 3], for recent review of such models see [4]). These values 
are generally very difficult to obtain in classical skin penetration experiments [5], as well as in less common skin desorption experiments $[6,7]$, due to difficulties in assessing the true path-length of solute transport through the SC [8]. In this work, we deployed the technique of Fluorescence Recovery After Photobleaching (FRAP) [9, 10] realised on a fluorescence multiphoton tomography (FMT) to probe diffusion of organic fluorescent dye Rhodamine B $(\mathrm{Rh}: \mathrm{B}, \mathrm{MW}=479, \log \mathrm{P}=1.95)$ in the SC lipid phase. FRAP implemented by means of FMT allows is capable to realise dimensions of the photo-bleached volume from sub-micron to sub-millimetre in linear dimention. Therefore, this volume can be localised fully within the lipid phase of the SC, allowing determination of the value of local diffusion coefficient. Although FRAP has been used before to assess diffusion coefficients of various fluorescent probes in artificial lipid bilayer systems [11], to our knowledge, the presented technique for the first time determines the diffusion coefficient of a solute in the SC lipid phase using FRAP. Since the artificial lipid phases can potentially have structure and composition different to those of the lipid phase of SC, we believe that this addition to the experimental toolbox of SC transport studies is a significant development. Furthermore, the proposed technique can be further developed, by appropriate choice of a fluorophore and by a proper selection of site of the photobleaching, to study the transport inside corneocytes and across a corneocyte envelope. This development has a potential to further advance the understanding of relative importance of different pathways $[7,12,13]$ on the overall solute transport across SC.

\section{Methods}

All experiments were performed using FMT based on Zeiss510 (Zeiss, Germany) system with a water-immersion objective lens $(63 \times$, N.A. 1.3). Zeiss510 system operated in combination with Mai Tai XF-1 femtosecond pulsed laser with a wavelength tuning range from 710 to $920 \mathrm{~nm}$. The pulse width was evaluated as $<100 \mathrm{fs}$ based on the spectral bandwidth measurement at $80-\mathrm{MHz}$ repetition rate, resulting in the high instantaneous peak power $\left(\sim 100 \mathrm{GW} / \mathrm{cm}^{2}\right)$ at the focal spot. A femtosecond laser operated at a centre wavelength of $810 \mathrm{~nm}$ with power incident at the sample estimated as 33 or $16.5 \mathrm{~mW},(1 \%$ or $0.5 \%$ of the total laser output energy which was $3310 \mathrm{~mW}$ ) was employed as the excitation source. To achieve the photobleaching regime, the laser power was increased to $165 \mathrm{~mW}$. A bandpass filter centred at a wavelength of $560 \mathrm{~nm}$ (bandwidth, $60 \mathrm{~nm}$ ) was used to pass the Rh:B fluorescence to a detector. 
Glycerol experiments. Since SC is a complex, highly heterogeneous structure, it was necessary, first, to establish the FRAP method, including optimisation of various experimental parameters, using a simplified model of homogeneously distributed fluorescence. Rh:B solution in glycerol was chosen as this homogeneous fluorescence model, because glycerol possesses high viscosity that was comparable to that of the skin lipids. This model was also preferred, as it has been described in the literature [14]. Experiments with glycerol solutions of Rh:B were first performed to establish the depth of the photobleached volume. This depth was determined from the axial point spread function (axial PSF) of the FMT used in all FRAP experiments. In order to determine the axial PSF, a 10- $\mu \mathrm{L}$ sample of $10-\mu \mathrm{M} R \mathrm{Rh}: \mathrm{B}$ in glycerol solution sealed in an o-ring container on a glass slide was scanned at the boundary of the sample and the glass slide. The resultant fluorescent intensity versus depth curve was then derived to obtain the axial PSF curve. The full width at half maximum (FWHM) of the axial PSF was interpreted as the depth of the photo-bleached volume. The axial PSF was dependent mainly on the N.A. of the microscope objective lens assuming its aberration-free characteristics. FRAP experiments were performed with the $10-\mu \mathrm{M} R \mathrm{Rh}: \mathrm{B}$ in glycerol samples. The samples of the $\mathrm{Rh}: \mathrm{B}$ solution in glycerol were positioned between glass slide and the cover slip so that the sample was at least $150-\mu \mathrm{m}$ thick. Photobleached volume of cylindrical shape was formed in the sample to be located far from the boundaries, close to the middle of the sample. The diameter of the cylindrical photobleached volume was chosen to be of similar dimension to the determined depth of photobleaching. The photobleaching was realised by scanning the volume to be photobleached by gradually increasing the laser power $(165,330$ or $495 \mathrm{~mW})$. Scanning the tissue with the high laser power results in permanent destruction of fluorescence (photobleaching) of Rh:B molecules in the photobleached volume. After the photobleaching stops, $\mathrm{Rh}: \mathrm{B}$ molecules from nonphotobleached areas of the sample start to diffuse into the photobleached volume, thus recovering the fluorescent molecule concentration in the volume. The recovery of the fluorescence in the photobleached volume was measured by scanning with a reduced laser power $(33 \mathrm{~mW})$.

FRAP experiment in SC. Pieces of human skin were obtained from one subject and SC prepared using trypsin treatment of heat-separated epidermis, as previously described [15]. $\mathrm{SC}$ was soaked in $10-\mu \mathrm{M} \mathrm{Rh}: \mathrm{B}$ solution in water for two days in order to guarantee a steady state concentration distribution of the fluorophore throughout all SC phases. After saturation, 
the SC sample was removed from the solution and excess solution was wiped with tissue. Then SC was placed between a glass slide and a cover slip and sealed to prevent the drying of the sample. FRAP was conducted at different positions in the SC using the FMT. Significant $\mathrm{Rh}: \mathrm{B}$ fluorescence was detected only in the lipid phase of the SC, most likely due to the lipophilic nature of the fluorophore. The results for further mathematical analysis were selected by ensuring that the largest possible photobleached volume was all within the lipid phase of the SC. As with the glycerol FRAP experiments, photobleaching of SC was achieved by scanning the volume to be photobleached by gradually increasing the laser power $(165 \mathrm{~mW})$. The recovery of the fluorescence in the photobleached volume was measured by scanning with the reduced power $(16.5 \mathrm{~mW})$. Both circular and square photobleached areas were used in the SC FRAP experiments. Diameter of the circle and side of the square were selected to be of similar dimension to the depth of the photobleaching.

Mathematical models and data analysis. Two simple mathematical models are considered: the first (referred to as the 3D-model) is based on the solution of the diffusion equation with the assumption that the diffusion medium around the photobleached volume is infinite. The second model (referred to as the 2D-model) is based on the assumption that the fluorophore diffusion occurs only in the lipid phase of the SC and this phase is an infinite 2D-layer of thickness equal or less than the photobleached volume. This layer is limited by assumed impermeable corneocytes from the top and bottom (this assumption is mathematically identical to the assumption of no fluorophore present in the corneocytes). For both models, it is assumed that the photobleaching is uniform within the photobleached volume $\left(V_{P B}\right)$, that is, if $C(\mathbf{x}, t)$ is the fluorophore concentration at the time $t$ and at a position $\mathbf{x}=(x, y, z)$, then immediately after photobleaching occurs, the concentration is:

$$
C(\mathbf{x}, 0)=\left\{\begin{array}{cc}
\alpha C_{0} & \text { inside } V_{P B} \\
C_{0} & \text { outside } V_{P B}
\end{array}\right.
$$

where $C_{0}$ is the original concentration of the fluorophore and $0 \leq \alpha<1$ is the degree of photobleaching. The concentration at any time after the photobleaching can be found by solving the diffusion equation (2) with initial condition (1).

$$
\frac{\partial C(\mathbf{x}, t)}{\partial t}=D \Delta C(\mathbf{x}, t)
$$

where $D$ is the diffusion coefficient. The total fluorescence inside the $V_{P B}(F(t))$ can be found as: 


$$
F(t)=\oiiint_{V_{P B}} C(\mathbf{x}, t) d^{3} \mathbf{x}
$$

127 where we assumed that fluorescence intensity is proportional to the concentration of the

128 fluorophore. 3D-model is assumed in eq. 3, for 2D-model, the triple integral in eq. 3 and 129 integrals below need to be replaced with double integrals.

130

131 The initial condition for the problem can be simplified by a change of function, so that:

$$
C_{f}(\mathbf{x}, t)=C_{0}-C(\mathbf{x}, t)
$$

132 so that initial condition for $C_{f}$ is:

$$
C_{f}(\mathbf{x}, 0)=\left\{\begin{array}{cc}
(1-\alpha) C_{0} & \text { inside } V_{P B} \\
0 & \text { outside } V_{P B}
\end{array}=(1-\alpha) C_{0} U(\mathbf{x})\right.
$$

133 where $U(\mathbf{x})$ is defined as:

$$
U(\mathbf{x})=\left\{\begin{array}{lc}
1 & \text { inside } V_{P B} \\
0 & \text { outside } V_{P B}
\end{array}\right.
$$

134 and $C_{f}$ satisfies the diffusion equation (2). The total fluorescence inside $V_{P B}$ can be defined 135 using $C_{f}$ :

$$
F(t)=V_{P B} C_{0}-\oiiint_{V_{P B}} C_{f}(\mathbf{x}, t) d^{3} \mathbf{x}=V_{P B} C_{0}-\int_{-\infty}^{\infty} \int_{-\infty}^{\infty} \int_{-\infty}^{\infty} U(\mathbf{x}) C_{f}(\mathbf{x}, t) d x d y d z
$$

136

137 Taking Fourier transform of the diffusion equation and initial condition then solving yields:

$$
\hat{C}_{f}(\mathbf{k}, t)=C_{0}(1-\alpha) \hat{U}(\mathbf{k}) \exp \left(-\mathbf{k}^{2} t\right)
$$

138 where

$$
\hat{C}_{f}(\mathbf{k}, t)=\int_{-\infty}^{\infty} \int_{-\infty}^{\infty} \int_{-\infty}^{\infty} C_{f}(\mathbf{x}, t) \exp (i \mathbf{k x}) d x d y d z
$$

139 is the Fourier transform of $C_{f}$, and

$$
\hat{U}(\mathbf{k})=\int_{-\infty}^{\infty} \int_{-\infty}^{\infty} \int_{-\infty}^{\infty} U(\mathbf{x}) \exp (i \mathbf{k} \mathbf{x}) d x d y d z=\oiiint_{V_{P B}} \exp (i \mathbf{k} \mathbf{x}) d^{3} \mathbf{x}
$$

140 is the Fourier transform of $U$.

141

142 Using Parseval's theorem for Fourier transform: 


$$
\int_{-\infty}^{\infty} \int_{-\infty}^{\infty} \int_{-\infty}^{\infty} f(\mathbf{x}) g(\mathbf{x}) d x d y d z=\frac{1}{(2 \pi)^{3}} \int_{-\infty}^{\infty} \int_{-\infty}^{\infty} \int_{-\infty}^{\infty} \overline{\hat{f}(\mathbf{k})} \hat{g}(\mathbf{k}) d k_{1} d k_{2} d k_{3}
$$

143

in the integral on right hand side of eq. (7) yields:

$$
\int_{-\infty}^{\infty} \int_{-\infty}^{\infty} \int_{-\infty}^{\infty} U(\mathbf{x}) C_{f}(\mathbf{x}, t) d x d y d z=\frac{1}{(2 \pi)^{3}} \int_{-\infty}^{\infty} \int_{-\infty}^{\infty} \int_{-\infty}^{\infty} \overline{\hat{U}(\mathbf{k})} \hat{C}_{f}(\mathbf{k}) d k_{1} d k_{2} d k_{3}
$$

144 together with eq. (8) this gives:

$$
\int_{-\infty}^{\infty} \int_{-\infty}^{\infty} \int_{-\infty}^{\infty} U(\mathbf{x}) C_{f}(\mathbf{x}, t) d x d y d z=\frac{C_{0}(1-\alpha)}{(2 \pi)^{3}} \int_{-\infty}^{\infty} \int_{-\infty}^{\infty} \int_{-\infty}^{\infty}|\hat{U}(\mathbf{k})|^{2} \exp \left(-\mathbf{k}^{2} t\right) d k_{1} d k_{2} d k_{3}
$$

145 Therefore, the total fluorescence inside $V_{P B}$ can be found as:

$$
F(t)=V_{P B} C_{0}-\frac{C_{0}(1-\alpha)}{(2 \pi)^{3}} \int_{-\infty}^{\infty} \int_{-\infty}^{\infty} \int_{-\infty}^{\infty}|\hat{U}(\mathbf{k})|^{2} \exp \left(-\mathbf{k}^{2} t\right) d k_{1} d k_{2} d k_{3}
$$

146 with $\hat{U}(\mathbf{k})$ defined in eq. (10).

147

148 For the cylindrical $V_{P B}$ (3D-model) eq. (13) yields:

$$
\begin{gathered}
F(t)=\frac{\pi d^{2} h C_{0}}{4}\left\{1-(1-\alpha)\left[1-e^{-d^{2} /(8 D t)}\left(I_{0}\left(\frac{d^{2}}{8 D t}\right)+I_{1}\left(\frac{d^{2}}{8 D t}\right)\right)\right] \times\right. \\
\left.\left[\operatorname{erf}\left(\frac{h}{2 \sqrt{D t}}\right)+\sqrt{\frac{4 D t}{\pi h^{2}}}\left(e^{-h^{2} /(4 D t)}-1\right)\right]\right\}
\end{gathered}
$$

149 where $d$ is the diameter and $h$ is the depth of the photobleached volume, respectively.

150

151 For the square prism $V_{P B}$ (3D-model) eq. (13) yields:

$$
\begin{gathered}
F(t)=a^{2} h C_{0}\left\{1-(1-\alpha)\left[\operatorname{erf}\left(\frac{a}{2 \sqrt{D t}}\right)+\sqrt{\frac{4 D t}{\pi a^{2}}}\left(e^{-a^{2} /(4 D t)}-1\right)\right]^{2} \times\right. \\
\left.\left[\operatorname{erf}\left(\frac{h}{2 \sqrt{D t}}\right)+\sqrt{\frac{4 D t}{\pi h^{2}}}\left(e^{-h^{2} /(4 D t)}-1\right)\right]\right\}
\end{gathered}
$$

152 where $a$ is the side of the square and $h$ is the depth of the photobleached volume, 153 respectively.

155 For the 2D-model equations for the total fluorescence are derived by considering double, instead of triple integrals in eqs. (10), and (13) yielding: 


$$
\begin{aligned}
& F(t)=\frac{\pi d^{2} C_{0}}{4}\left\{1-(1-\alpha)\left[1-e^{-d^{2} /(8 D t)}\left(I_{0}\left(\frac{d^{2}}{8 D t}\right)+I_{1}\left(\frac{d^{2}}{8 D t}\right)\right)\right]\right\} \\
& F(t)=a^{2} C_{0}\left\{1-(1-\alpha)\left[\operatorname{erf}\left(\frac{a}{2 \sqrt{D t}}\right)+\sqrt{\frac{4 D t}{\pi a^{2}}}\left(e^{-a^{2} /(4 D t)}-1\right)\right]^{2}\right\}
\end{aligned}
$$

157 for the circular and square photobleaching areas, respectively.

159 The total fluorescence intensity recovery data was fitted to mathematical models using

160 Scientist software (MicroMaths Scientific Software).

\section{Results and Discussion}

163 Examples of the axial PSF are presented in Fig. 1. The analysis of this data yielded the depth 164 of the photobleaching volume of $2.7 \pm 0.3 \mu \mathrm{m}$. In FRAP experiments, the lateral dimensions 165 of the photobleached volume were selected to be close to the depth of a photobleached volume.

FRAP experiments for $\mathrm{Rh}: \mathrm{B}$ in glycerol were performed using a cylindrical photobleached volume of diameter $\approx 2.5 \mu \mathrm{m}$. The fluorescence intensity recovery data were fitted using eq. 14 , resulting in the diffusion coefficient of $D=(3.2 \pm 0.5) \times 10^{-9} \mathrm{~cm}^{2} \mathrm{~s}^{-1}(\mathrm{n}=3)$. The value of the diffusion coefficient of Rh:B in glycerol can also be approximated using empirical Wilke-

172 Chang relationship:[16]

$$
D=7.4 \times 10^{-8} \frac{T M^{1 / 2}}{\eta V^{0.6}}
$$

173

where $D$ is in $\mathrm{cm}^{2} \mathrm{~s}^{-1}, T$ is the temperature $\left(=298^{\circ} \mathrm{K}\right)$ at which the diffusion coefficient is measured, $M$ is the molecular weight of the glycerol $\left(92 \mathrm{~g} \mathrm{~mole}^{-1}\right)$ and $\eta$ is the dynamic viscosity of glycerol (934 centipoise, from Wolfram Alpha). Further, $V$ is the molar volume of Rh:B at the normal boiling point, which was estimated as $M W / \rho$, where $\rho\left(\approx 1 \mathrm{~g} \mathrm{~cm}^{-3}\right.$, from Wolfram Alpha), and $M W\left(=479 \mathrm{~g} \mathrm{~mole}^{-1}\right)$ is the molecular weight of the Rh:B. Using these values in eq. 18 , yields $D \approx 5.6 \times 10^{-9} \mathrm{~cm}^{2} \mathrm{~s}^{-1}$, which is reasonably close to the value obtained from the fitting of the FRAP data $\left(3.2 \times 10^{-9} \pm 0.5 \mathrm{~cm}^{2} \mathrm{~s}^{-1}\right)$, considering that eq. 18 is only an approximation. It can therefore be concluded that the FRAP experiments of the Rh:B in glycerol yielded reasonable value for the diffusion coefficient. 
In Fig. 2, Z-stack FMT image set of the SC is presented, with images (a) to (e) acquired from closer to the surface of the SC to more in-depth layers of the SC at an interval of $1 \mu \mathrm{m}$. In images (b) to (d), a circular photobleached area is apparent, consistent with the cylindrical photobleached volume. The 3D-data from the Z-stack FMT image sets similar to that presented in Fig. 2 allows determination of the local structure of the SC phases (corneocytes and lipids) around the photobleached volume. For example, it can be seen in Fig 2 that corneocytes appear as areas of little $\mathrm{Rh}: \mathrm{B}$ fluorescence and lipid phase as bright $\mathrm{Rh}: \mathrm{B}$ fluorescence. In principle, using this information it would be possible to construct a numerical finite-element model of the SC for each individual instance of FRAP experiment. While we understand that this type of mathematical modelling would represent a gold standard, in this work, we consider it appropriate to make simplifying assumptions that would give an analytical solution for the total fluorescence. We based the first mathematical model for the SC FRAP experiments on the assumption that the diffusion medium is infinite (referred to as the 3D-model). This assumption would have been valid, if the diffusion coefficient, as well as solubility, were the same in both the lipid and corneocyte phases of the SC. The second model is based on the assumption that diffusion occurs only in the lipid phase of the SC, and this phase is structured as an infinite layer sandwiched between two corneocyte layers (referred to as the 2D-model). This solution approximates the situation when corneocytes are impermeable to $\mathrm{Rh}: \mathrm{B}$ or the concentration of $\mathrm{Rh}: \mathrm{B}$ in them is negligible (due to, for example, low solubility). The real scenario is probably somewhere in between these two extreme cases, but given the fact that corneocytes have little Rh:B fluorescence (see Fig. 2), possibly closer to that of the 2D-model.

The square prism photobleached volume was used in SC FRAP experiments with the side of the square set to $2 \mu \mathrm{m}$. An example of the data of the total fluorescence versus time and its regressions with the 2D- and 3D- models is shown in Fig 3. It can be seen that the quality of the regression is very good for both 2D- and 3D- models, which yield very similar curves.

210 The value of the diffusion coefficient estimated by fitting the 3D-model (eq. 15) to the 211 experimental data $(\mathrm{n}=6)$ was $3.5 \pm 1.0 \times 10^{-9} \mathrm{~cm}^{2} \mathrm{~s}^{-1}$. The 2D-model (eq. 17) yielded the 212 diffusion coefficient of $6.3 \pm 1.7 \times 10^{-9} \mathrm{~cm}^{2} \mathrm{~s}^{-1}$. It can be noted that the values for the two 213 distinct models differ by less than a factor of 2, yielding reasonably narrow range for the 214 value of the diffusion coefficient of $\mathrm{Rh}: \mathrm{B}$ in SC lipid phase. Both of these values are 215 substantially less than the value of the diffusion coefficient for a similar molecular weight 216 fluorophore (4a-diaza-s-indacene-3-hexadecanoic acid, MW=474) found by Johnson et al 
$217[11]\left(D=19.2 \pm 1.7 \times 10^{-9} \mathrm{~cm}^{2} \mathrm{~s}^{-1}\right)$. In their work, the FRAP technique was used to measure a

218 solute diffusion coefficient in artificial lipid bi-layers prepared from a dimyristoyl-

219 phosphatidylcholine/cholesterol (40 mol\%) mixture. This difference in values for the

220 diffusion coefficients could be in part due to the higher temperature of the authors'

221 experiments: $27^{\circ} \mathrm{C}$, compared to our experiments conducted at room temperature $\left(22-25^{\circ} \mathrm{C}\right)$.

222 The value of the diffusion coefficient can be influenced by the lipid-fluorophore molecular

223 interactions, which are different for two fluorophores considered. Another possibility is that

224 the structure of natural SC lipid bi-layers slows diffusion compared to that of the artificial

225 lipid bi-layers used in the experiments reported by Johnson et al [11].

226

227 Since corneocytes appear as areas of little Rh:B fluorescence (see Fig. 2), FRAP experiments

228 in the corneocyte phase of the SC have not yielded useful data. It is expected, though, that an

229 appropriate selection of fluorophore, exhibiting measurable fluorescence in the corneocyte

230 phase should allow determination of the value of the diffusion coefficient in this phase. It can

231 be also argued that if coenocytes are not permeable or have little fluorophore present

232 (corresponding to 2D-model case) the diffusion measured by the presented FRAP technique

233 is the lateral diffusion in the lipid layers. Indeed, due to lipid layers orientation (parallel to

234 corneocyte's surface) diffusion in these layers will happen in the lateral direction only

235 (sandwiched between two corneocytes above and below) and, only the lateral diffusion in the

236 lipid layers is likely to be measured by this technique. If fluorophore can be selected so that it

237 is present in the coenocyte as well as corneocyte's wall being permeable to the fluorophore

238 (this corresponds to our 3D-model), then molecular transport above and below the photo-

239 bleached volume will be trans-lipid layer diffusion (as well as trans corneocyte wall), and

240 should allow determination of the trans-lipid layer diffusion coefficient as well.

\section{Conclusion}

243 A FRAP method for determining local values of diffusion coefficient in SC was developed.

244 The method was, first, verified using the simplified system of $\mathrm{Rh}: \mathrm{B}$ in glycerol solution

245 followed by application to human SC. For Rh:B fluorophore in SC FRAP it was found that

246 only the diffusion coefficient in SC lipids can be determined from SC FRAP experiments. It

247 was hypothesised that extending the SC FMT FRAP method to different fluorophores can

248 yield local diffusion coefficients in both lipid and corneocyte phases. 


\section{Acknowledgments}

251 We acknowledge the support by the NHMRC of Australia. We are grateful to Dr Tony O'Connor for discussions on applying Parseval's theorem to obtain analytical expression for the fluorescence recovery function.

\section{Figure legends}

Fig 1. Examples of the axial PSFs obtained by using a $63 \times$, N.A. 1.3, water-immersion objective lens at six different sites.

Fig 2. Z-stack FMT image set of the SC with a cylindrical photobleaching volume evident as a dark circle pointed by a white arrow in image (c). Images (a) to (e) are from closer to the surface of the SC to more in-depth layers of the SC at an interval of $1 \mu \mathrm{m}$. Scale bar is $30 \mu \mathrm{m}$.

Fig 3. Total fluorescence vs. time data for SC (squares) and its regressions with the 2D (dashed line) and 3D models (solid line).

\section{References}

267 [1] Y. Wu, Y.G. Anissimov, M.S. Roberts, Introduction to dermatokinetics, in: S.N. Murthy

268 (Ed.) Dermatokinetics of Therapeutic Agents, CRC Press Boca Raton, 2011, pp. 1-23. [2] T.F. Wang, G.B. Kasting, J.M. Nitsche, A multiphase microscopic diffusion model for stratum corneum permeability. I. Formulation, solution, and illustrative results for representative compounds, J. Pharm. Sci., 95 (2006) 620-648.

[3] T.F. Wang, G.B. Kasting, J.M. Nitsche, A multiphase microscopic diffusion model for stratum corneum permeability. II. Estimation of physicochemical parameters, and application to a large permeability database, J. Pharm. Sci., 96 (2007) 3024-3051. [4] S. Mitragotri, Y.G. Anissimov, A.L. Bunge, H.F. Frasch, R.H. Guy, J. Hadgraft, G.B. Kasting, M.E. Lane, M.S. Roberts, Mathematical models of skin permeability: An overview, Int. J. Pharm., (2011). [5] M.S. Roberts, Y.G. Anissimov, Mathematical models in percutaneous absorption, in: R.L. Bronaugh, H.I. Maibach (Eds.) Percutaneous Absorption Drugs -- Cosmetics -- Mechanisms - Methodology, Marcel Dekker, New York, 2005, pp. 1--44.

281 [6] Y.G. Anissimov, M.S. Roberts, Diffusion modeling of percutaneous absorption kinetics: 3. Variable diffusion and partition coefficients, consequences for stratum corneum depth profiles and desorption kinetics, J. Pharm. Sci., 93 (2004) 470-487. Desorption Kinetics., J. Pharm. Sci., 98 (2009) 772-781. 
287 [8] A.L. Bunge, R.H. Guy, J. Hadgraft, The determination of a diffusional pathlength through

288 the stratum corneum, Int. J. Pharm., 188 (1999) 121-124.

289 [9] K. Braeckmans, L. Peeters, N.N. Sanders, S.C. De Smedt, J. Demeester, Three290 dimensional fluorescence recovery after photobleaching with the confocal scanning laser 291 microscope, Biophys. J., 85 (2003) 2240-2252.

292 [10] Y.H. Sniekers, C.C. van Donkelaar, Determining diffusion coefficients in 293 inhomogeneous tissues using fluorescence recovery after photobleaching, Biophys. J., 89 294 (2005) 1302-1307.

295 [11] M.E. Johnson, D.A. Berk, D. Blankschtein, D.E. Golan, R.K. Jain, R.S. Langer, Lateral 296 diffusion of small compounds in human stratum corneum and model lipid bilayer systems, 297 Biophys. J., 71 (1996) 2656-2668.

298 [12] G.B. Kasting, N.D. Barai, T.F. Wang, J.M. Nitsche, Mobility of water in human stratum 299 corneum, J. Pharm. Sci., 92 (2003) 2326-2340.

300 [13] J.C. Keister, G.B. Kasting, Use of transient diffusion to investigate transport pathways 301 through skin, J. Controlled Release, 4 (1986) 111-117.

302 [14] J. Braga, J.M. Desterro, M. Carmo-Fonseca, Intracellular macromolecular mobility 303 measured by fluorescence recovery after photobleaching with confocal laser scanning 304 microscopes, Mol. Biol. Cell, 15 (2004) 4749-4760.

305 [15] A.M. Kligman, E. Christophers, Preparation of isolated sheets of human stratum 306 corneum, Arth Dermatol, 88 (1963) 702-705.

307 [16] C.R. Wilke, P. Chang, Correlation of Diffusion Coefficients in Dilute Solutions, Aiche J, 3081 (1955) 264-270. 


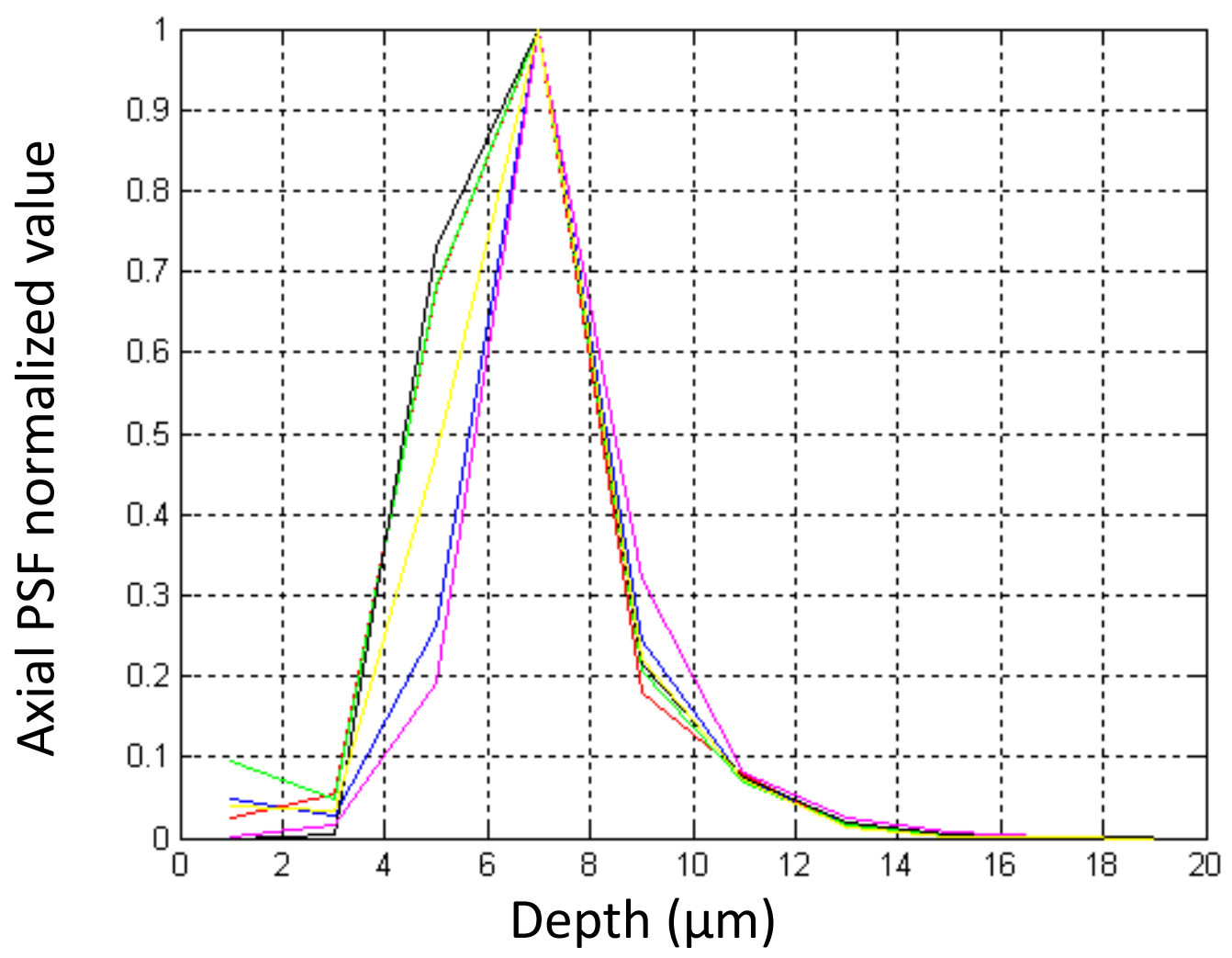

Fig 1. Examples of axial PSF for 63X, 1.0NA, water immersion objective lens at six different sites. 
(a)

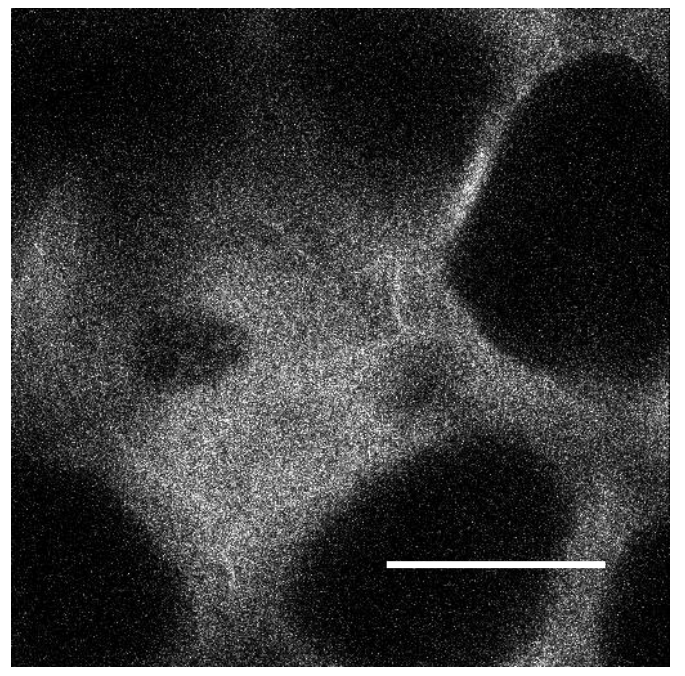

(d)

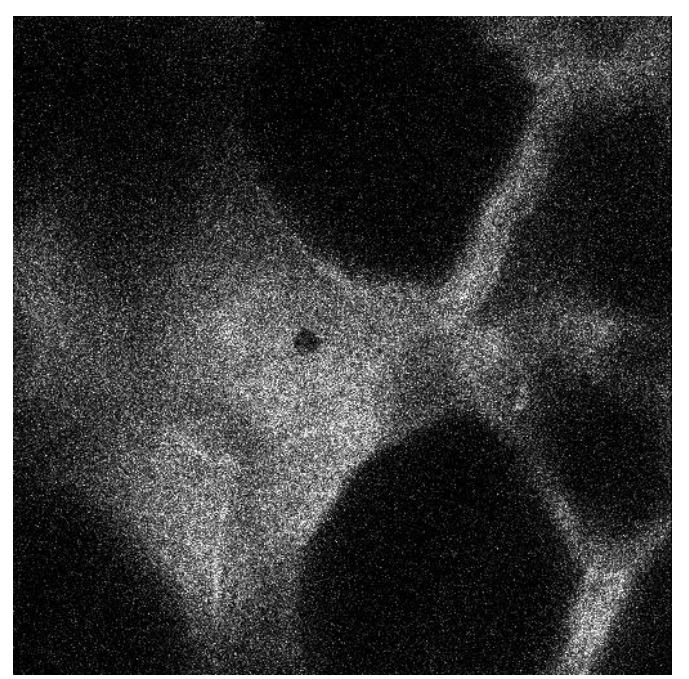

(b)

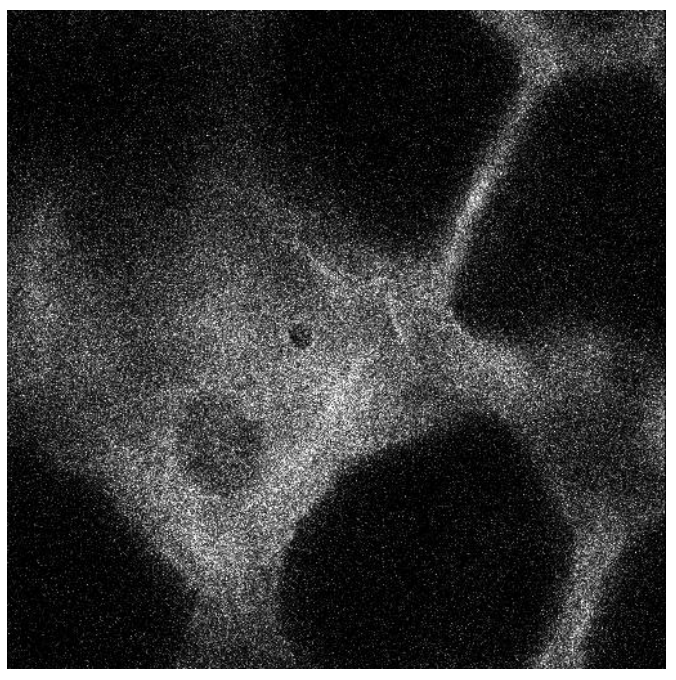

(e) (c)

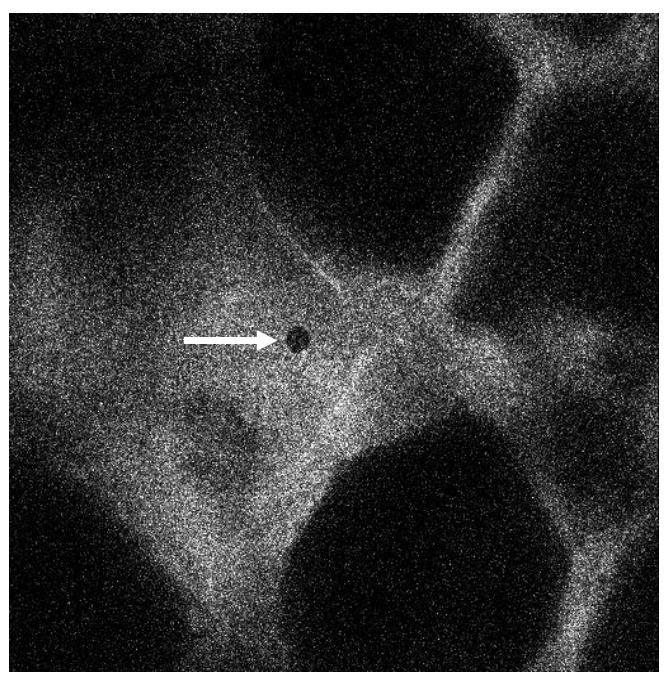

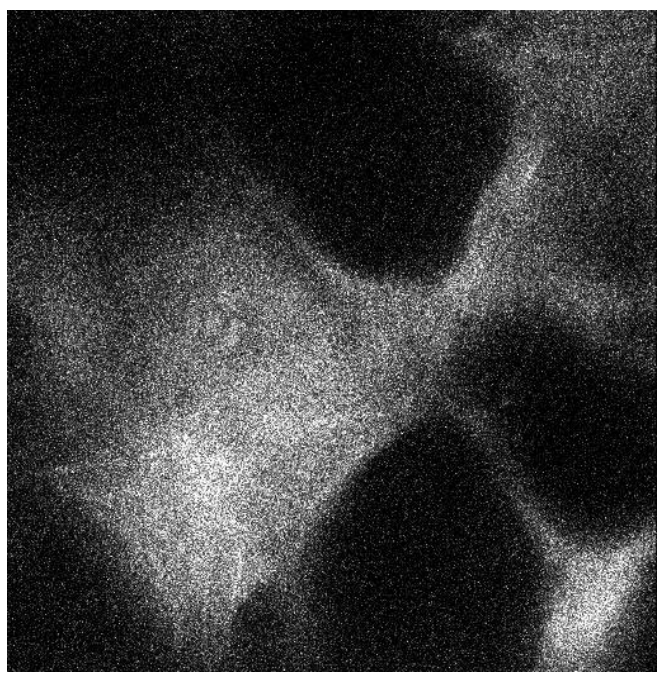

Fig 2. Z-stack FMM image set of the SC with a cylindrical photo-bleaching volume evident as a dark circle pointed by a white arrow in image (c). Images (a) to (e) are from closer to the surface of the SC to more in-depth layers of the SC at an interval of $1 \mu \mathrm{m}$. Scale bar is $30 \mu \mathrm{m}$. 


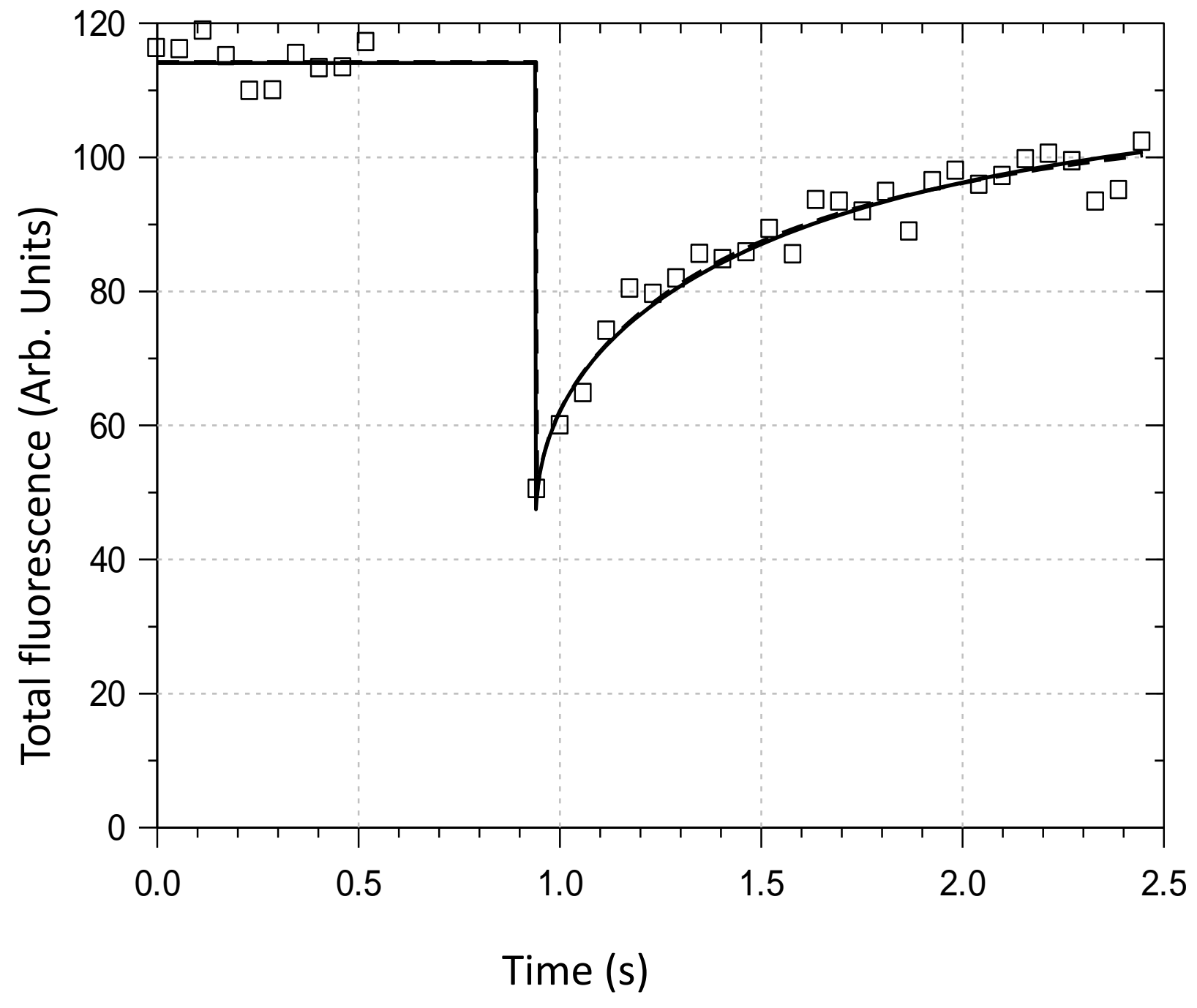

Fig 3. Total fluorescence vs. time data for SC (squares) and its regressions with the $2 \mathrm{D}$ (dashed line) and 3D models (solid line). 
SC photo-bleaching

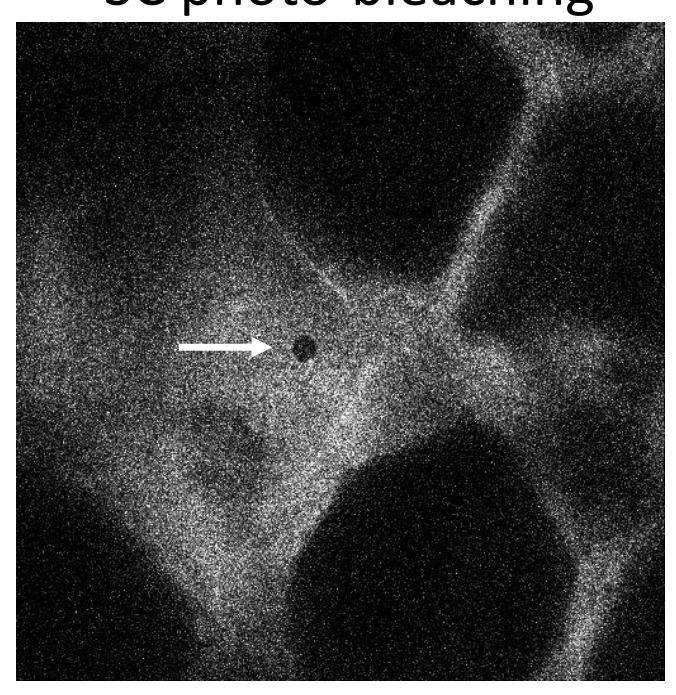

SC FRAP

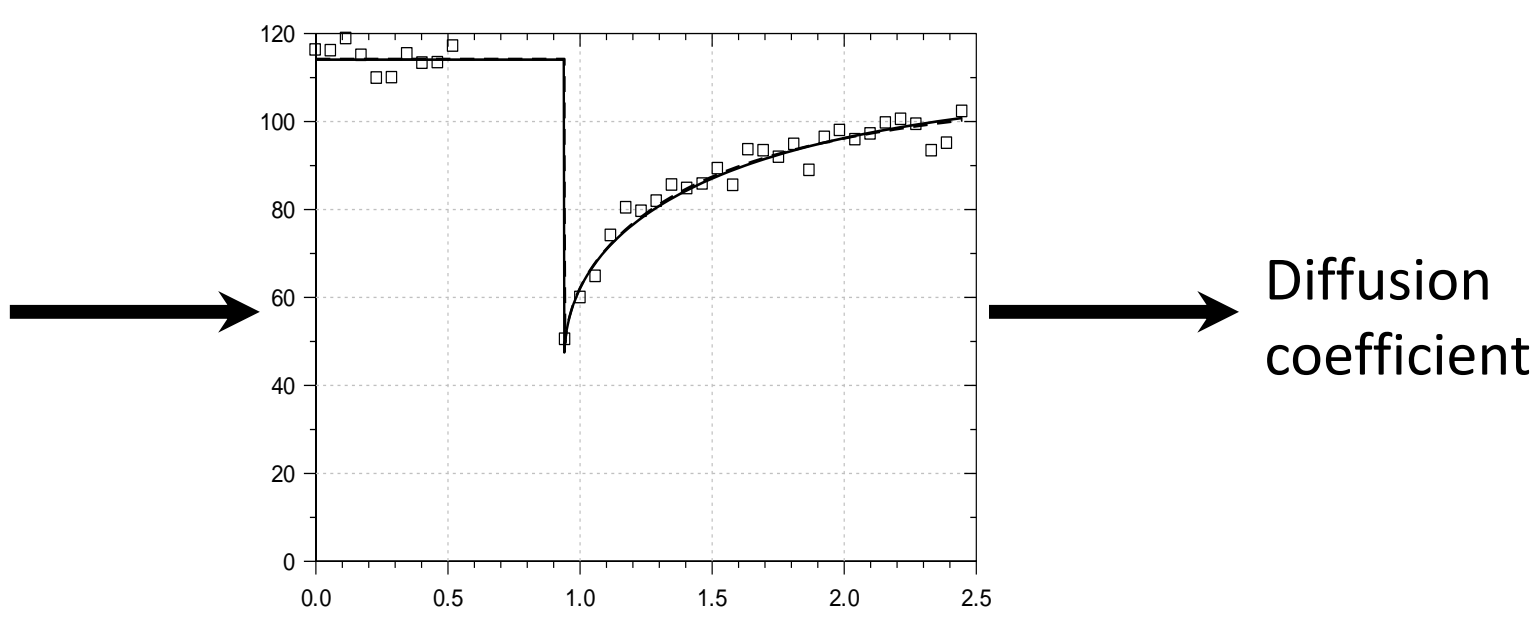

